\title{
Desarrollo y validación del Test de Ansiedad Social para estudiantes universitarios (TAS-U)
}

\section{Development and validation of the Social Anxiety Test in university students (SAT-U)}

\author{
Valeria E. Moran a,b,*, Fabián O. Olaz ${ }^{\mathrm{b}}$ Edgardo R. Pérez ${ }^{\mathrm{b}}$, Zilda A. P. Del Prette

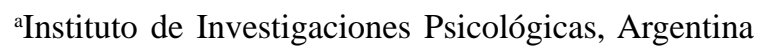 \\ bUniversidad Nacional de Córdoba, Argentina \\ 'Universidade Federal de São Carlos, Brasil
}

\section{Resumen}

El estudio de la ansiedad social en estudiantes universitarios ha cobrado una especial relevancia considerando sus implicancias en el ajuste social y el bienestar psicológico de quienes la presentan. A pesar de ello, no existen instrumentos de evaluación que estén dirigidos específicamente a esta población, quienes cotidianamente se enfrentan a situaciones particulares que pueden ser potencialmente generadoras de ansiedad. Se presenta el Test de Ansiedad Social para estudiantes universitarios (TAS-U) aportando evidencias de validez y confiabilidad. Se diseñaron 68 ítems preliminares teniendo en cuenta literatura, escalas de antecedentes y consultas con expertos. Utilizando muestreo no probabilístico, se realizaron análisis factoriales exploratorios y confirmatorios de una muestra de estudiantes universitarios argentinos. Se obtuvo una escala final compuesta por 27 ítems distribuidos en cuatro factores (CFI $=.95$, TLI $=.95$, RMSEA $=.063 \mathrm{y}$ WRMR = 1.19). Sobre la consistencia interna de la escala, los coeficientes alfa de Cronbach y de fiabilidad compuesta fueron buenos y excelentes para cada factor. Las dimensiones son coincidentes con factores evidenciados en estudios de otras escalas similares y con postulados teóricos conceptuales sobre ansiedad social.

Palabras clave: ansiedad social, estudiantes universitarios, medición, validación.

Para citar este artículo:

Moran, V., Olaz, F., Pérez, E., \& Del Prette, Z. (2018). Desarrollo y validación del Test de Ansiedad Social para estudiantes universitarios (TAS-U). Liberabit, 24(2), 195-212. https://doi.org/10.24265/ liberabit.2018.v24n2.03

\begin{abstract}
The study of social anxiety in college students has gained particular importance if we consider its role in their social adjustment and psychological well-being. Despite this, there are no assessment tools that are specifically aimed at this population, which face events that can potentially generate anxiety on a daily basis. The Social Anxiety Test for University students (SAT-U) is presented herein with evidence of validity and reliability. Sixty-eight (68) preliminary items were designed taking into account the current literature, previous scales and consultation with experts. By using a non-probabilistic sampling, exploratory and confirmatory factorial analyses were performed in a sample of Argentinian college students. A final scale was obtained with 27 items distributed in four factors (CFI = .95, TLI = .95, RMSEA $=.063$ and WRMR = 1.19). Regarding the internal consistency of the scale, Cronbach's alpha coefficients and the coefficient of composite reliability were good and excellent for each factor. The dimensions coincide with factors seen in studies with other similar scales, and with conceptual and theoretical postulates about social anxiety.
\end{abstract}

Keywords: social anxiety, university students, assessment, validation.

Este es un artículo Open Access bajo la licencia Creative Commons Atribución-NoComercial-CompartirIgual 4.0

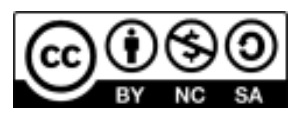




\section{Introducción}

Si bien experimentar ansiedad ante determinadas situaciones de interacción social resulta común y habitual para todas las personas, la ansiedad social (AS) como entidad nosológica se constituye como tal de acuerdo a su significancia clínica y teniendo en cuenta en qué medida existe una interferencia negativa en la vida cotidiana y en las principales áreas vitales de quien la padece. La AS se caracteriza por miedo a la evaluación negativa en situaciones sociales de interacción o exposición en público, generando evitación o resistencia hacia ellas (American Psychiatric Association [APA], 2013). Surge ante la posibilidad de una evaluación interpersonal en situaciones sociales reales o imaginarias, es decir, es el producto de una preocupación del individuo sobre cómo es percibido y evaluado por los demás (Leary \& Kowalski, 1995). Esta preocupación involucra pensamientos y cogniciones negativas, acompañada de síntomas físicos, tales como sudoración, ruborización y aceleración del ritmo cardiaco.

Sumado al hecho de que es uno de los problemas interpersonales que más afecta a las personas a nivel mundial con una prevalencia anual de aproximadamente 7\% (Bandelow \& Michaelis, 2015), diversos estudios han mostrado altos niveles de comorbilidad entre la ansiedad social y una serie de trastornos como la depresión (Adams, Balbuena, Meng, \& Asmundson, 2016) y trastornos por abuso de sustancias (Nicholls, Staiger, Williams, Richardson, \& Kambouropoulos, 2014). Además, se ha encontrado evidencia de que la AS está asociada con un mayor riesgo a padecer otros problemas emocionales, lo que sugiere que la ansiedad social podría ser un factor de riesgo para el desarrollo de otras patologías o la existencia de factores etiológicos en común (Rapee \& Spence, 2004). Precisamente, estudios recientes dan principal importancia a la desregulación emocional en la explicación de diversos trastornos psicopatológicos (Gross \& Jazaieri, 2014), siendo el trastorno de ansiedad social uno de los más explicados por este factor (Morán, Olaz, Pérez, \& Del Prette, 2018).
Por otro lado, si bien los estudios epidemiológicos han demostrado consistentemente que la AS es padecida en mayor proporción por mujeres (Rana, Akhtar, \& Tahir, 2013), el curso del trastorno de AS es similar para ambos géneros y no hay estudios concluyentes sobre las diferencias en el deterioro funcional y la comorbilidad (Asher, Asnaani, \& Aderka, 2017).

Actualmente, los resultados de estudios epidemiológicos y descriptivos sobre AS también indican que la población de estudiantes universitarios es uno de los grupos más vulnerables (Black et al., 2015; Cummings, Caporino, \& Kendall, 2014; Delgado, Inglés, \& García-Fernández, 2013; Ham, Connolly, Milner, Lovett, \& Feldner, 2013). Por su parte, Agudelo-Vélez, Casadiegos-Garzón y SánchezOrtíz (2009) corroboraron los resultados obtenidos por Galli (2005) sobre la alta prevalencia de trastornos de ansiedad social en estudiantes universitarios y el efecto sobre el rendimiento, la deserción y el abandono académico, el bienestar emocional, entre otras variables.

La adolescencia es una fase evolutiva crítica y de gran vulnerabilidad que culmina cuando se produce la salida de la persona desde su núcleo familiar hacia la sociedad mayor. En la edad en la cual el individuo ingresa a una carrera superior (generalmente hacia los dieciocho o diecinueve años), se producen una serie de cambios de vital importancia tales como el abandono de la residencia familiar, la búsqueda de pareja y la búsqueda de trabajo; lo cual enfrenta al joven a diferentes demandas emocionales e interpersonales y lo exponen a nuevos entornos que pueden resultar amenazantes si la persona no cuenta con los recursos de afrontamiento necesarios. En este contexto, los estudiantes no solo están expuestos a la evaluación continua de docentes y autoridades, sino también de los miembros del grupo estudiantil al cual pertenecen (Velásquez et al., 2008).

Por todo esto, puede entenderse la alta prevalencia de problemas de funcionamiento social como así 
también de diversas patologías en esta población; lo cual explica, a su vez, el incremento de estudios empíricos y desarrollos teóricos surgidos en las últimas dos décadas. En este sentido, los resultados de diversas investigaciones indican una alta prevalencia de problemas clínicos y problemáticas interpersonales y sociales en estudiantes universitarios, tales como timidez (Ricker, Ridgeway, Eichele, Perry, \& Zimansky, 2018), ansiedad heterosexual y dificultades en la solución de problemas sociales (Collins, 2009), falta de asertividad (Parray \& Kumar, 2016), fracaso académico (Brook \& Willoughby, 2015), problemas conductuales y emocionales como depresión y ansiedad (Beiter et al., 2015), distintos tipos de adicciones (Walters, Bulmer, Troiano, Obiaka, \& Bonhomme, 2018), delincuencia y agresividad (Coker et al., 2015), problemas de pareja (Kaukinen, 2014) y problemas de familia (Raposa \& Hammen, 2018).

A pesar de la proliferación de estudios sobre este constructo en estudiantes universitarios a nivel global, en Argentina no se reportan investigaciones sobre ansiedad social. Posiblemente, debido a la ausencia de instrumentos de medición que permitan la interpretación válida y confiable de sus puntuaciones.

La medición de la ansiedad social es un campo que se ha desarrollado paralelamente al crecimiento de los estudios, cuyo interés se ha centrado en la comprensión y profundización del conocimiento sobre este trastorno. Dentro de las herramientas existentes, podemos encontrar instrumentos destinados a población clínica y numerosos dispositivos dirigidos a la población en general (Caballo et al., 2010; Connor et al., 2000; Heimberg, Mueller, Holt, Hope, \& Liebowitz, 1993; La Greca \& López, 1998; Heimberg et al., 1999; Turner, Stanley, Beidel, \& Bond, 1989).

Los instrumentos más utilizados a nivel internacional han establecido su estructura interna a partir de procesos hipotético-deductivos (Salazar, Caballo, \& Arias, 2016), basándose en los resultados del análisis factorial exploratorio (AFE) y confirmatorio (AFC) para determinar las dimensiones del constructo. Además, la mayoría de las interpretaciones de los factores han tenido fundamento en criterios clínicos (Fernández-Sogorb, Aparicio-Flores, Granados, Aparisi-Sierra, \& Inglés, 2018) establecidos por el DSM IV (APA, 2000). Las dimensiones de los instrumentos por un lado se han orientado a evaluar distintos aspectos cognitivos de la ansiedad determinados a partir de diversos estudios empíricos que han estudiado los factores etiológicos y de mantenimiento más relevantes (Wong \& Rapee, 2016). Por otro lado, otras escalas buscan medir con qué intensidad el fenómeno es padecido, por lo que cobra importante relevancia la pertinencia y la representatividad de las situaciones sociales que se contemplan de acuerdo a la población meta de evaluación, es decir, la adecuación contextual del contenido.

A modo de síntesis, las dimensiones que surgen en la mayoría de las escalas se vinculan con situaciones específicas generadoras de ansiedad, entre ellas la interacción social con conocidos y desconocidos, miedo a ser observado y miedo a la evaluación negativa (La Greca \& López, 1998; Heimberg et al., 1999). Los enunciados plantean escenarios y demandas generales sin dirigir a una población específica, como son los estudiantes universitarios, quienes cotidianamente se enfrentan a situaciones particulares que pueden ser potencialmente generadoras de ansiedad como, por ejemplo, rendir un examen oral o exponer un trabajo frente a la clase. Bhamani y Hussain (2012) argumentaron que contar con especificidad en la adecuación contextual de los instrumentos destinados a medir la ansiedad social en los estudiantes de educación superior sería una herramienta de suma importancia para los gabinetes de asistencia psicológica y consejería. Con este fin, construyeron una escala para universitarios de Pakistán, sobre la cual solo evaluaron los niveles de confiabilidad, sin reportar estudios de validez.

Teniendo en cuenta la importancia de contar con instrumentos que posean adecuadas propiedades psicométricas y que sean pertinentes a la población y 
su contexto, el objetivo del presente estudio es construir un instrumento de evaluación de ansiedad social dirigido específicamente para estudiantes universitarios.

\section{Método}

Para la construcción de la escala se utilizó un diseño de tipo instrumental (Montero \& León, 2002) compuesto por cinco estudios. En primer lugar, se redactaron los ítems y se aportó evidencia de validez de contenido mediante juicio de expertos. En segundo y tercer lugar, se constató la estructura interna de la escala mediante análisis factorial exploratorio y confirmatorio, respectivamente, y se analizó la consistencia interna. En cuarto lugar, se realizó un estudio de grupos contrastados según el sexo. Finalmente, se realizó un estudio de validez concurrente con variables teóricamente relacionadas.

\section{Participantes}

Para el estudio de juicio de expertos se seleccionaron mediante muestreo intencional a 6 profesionales expertos: 2 en construcción de escalas, 1 en construcción de pruebas de evaluación de ansiedad y 3 expertos en AS.

Las muestras utilizadas para los estudios estuvieron conformadas por estudiantes universitarios de 8 universidades de la provincia de Córdoba en Argentina: 25\% de la Universidad Siglo 21, 51\% de la Universidad Nacional de Córdoba, $12 \%$ de la Universidad Nacional de Río Cuarto, 8\% de la Universidad Cervantes y $4 \%$ distribuido en otras universidades. Las edades estuvieron comprendidas entre 18 y 30 años de edad, con predominio de tercer y cuarto año de cursado. Los participantes fueron seleccionados mediante muestreo no probabilístico accidental durante el horario de cursado. Además, se recolectaron los datos con el previo consentimiento de los participantes quienes fueron informados sobre su participación voluntaria, anónima y confidencial, y sobre los objetivos de la investigación.
Para realizar el análisis factorial exploratorio y el análisis de la consistencia interna de la escala, se trabajó con una muestra de 652 participantes, compuesta por $71.6 \%$ mujeres y $28.4 \%$ hombres (edad $M=21.88, D E=2.85$ ). Para el análisis factorial confirmatorio y el análisis de la fiabilidad compuesta, la muestra estuvo formada por 501 participantes compuesta por $73.5 \%$ mujeres y $26.5 \%$ hombres (edad $M=21.22, D E=2.63$ ). Para el análisis de evidencia concurrente, se utilizó una muestra de 440 participantes compuesta por $72 \%$ mujeres y $28 \%$ hombres (edad $M=21.3, D E=4.05$ ). Previo a los análisis, se evaluó la calidad de todas las bases de datos, analizando valores perdidos y casos atípicos univariados y multivariados.

\section{Instrumentos}

Test de Ansiedad Social para estudiantes universitarios (TAS-U). Para el diseño de los ítems preliminares, se analizaron dimensiones comunes y situaciones abordadas en cada uno de los ítems de 18 escalas de ansiedad social y fobia social, utilizadas en diferentes países y para diferentes poblaciones. Sumado a ello, se tuvieron en cuenta las consideraciones teóricas y empíricas sobre la ansiedad social, sus síntomas, escenarios y componentes. Finalmente, se revisaron dos escalas de evaluación de constructos relacionados con la interacción social (Morán, Olaz, \& Del Prette, 2015), construidos específicamente en nuestro medio para estudiantes universitarios con el fin de extraer las situaciones sociales, contextos y demandas comunes para esta población. Los ítems fueron redactados teniendo en cuenta situaciones sociales típicas en este tipo de problemática. Así, se contemplaron situaciones que generasen con mayor probabilidad AS según lo indicado por la literatura (APA, 2000; Beidel, Turner, \& Dancu, 1985; Eysenck, 2013) y entrevistas realizadas a expertos en la construcción de escalas de medición de este constructo (Loureiro, comunicación personal). Otros factores que se tuvieron en cuenta en la redacción de ítems fue la inclusión de diferentes tipos de desempeño (individual o en interacción), diversos 
contextos y demandas, distintos niveles de familiaridad de los participantes y también diferentes números de personas en la interacción. De esta manera, se obtuvieron 68 ítems iniciales con una escala de respuesta tipo Likert de 10 puntos (1 a 10) donde la persona que responde debe indicar la intensidad o el grado de tensión, nerviosismo o ansiedad que experimenta ante cada situación social planteada, por ejemplo, «Proponer una reunión o actividad a tu grupo de amigos».

Escala de Dificultades en la Regulación Emocional (DERS). Se utilizó la escala construida por Gratz y Roemer (2004) validada en estudiantes universitarios argentinos por Medrano y Trogolo (2016). Este instrumento evalúa déficits y dificultades en diferentes procesos involucrados en la regulación de las emociones, por ejemplo, «Cuando me enfado, pierdo el control sobre mis comportamientos». La versión local está compuesta por 21 ítems con un formato de respuesta Likert de 5 posiciones (desde 1 $=$ casi nunca, hasta 5 = casi siempre) y una estructura de cuatro factores: 1) dificultades en el control de impulsos (seis ítems), 2) falta de aceptación emocional (seis ítems), 3) interferencia en conductas dirigidas a metas (cinco ítems) y 4) falta de claridad emocional (cuatro ítems). En su análisis factorial confirmatorio, los autores evidenciaron la existencia de una estructura factorial de segundo orden, donde los cuatro factores mencionados se agrupaban para formar dos factores más denominados dificultades en el procesamiento emocional (agrupa los factores 2 y 4) y dificultades en la regulación de la respuesta emocional (agrupa los factores 1 y 3). Cabe señalar que las diferentes escalas, tanto de primero y segundo orden, presentan una elevada consistencia interna (valores $\alpha$ comprendidos entre .73 y .88).

\section{Procedimiento y análisis de datos}

Los ítems preliminares del instrumento fueron entregados a los jueces y se les solicitó que indicaran la pertinencia de cada ítem en base a su contenido y que los calificaran en sus aspectos formales (claridad semántica y corrección sintáctica) utilizando una escala de 1 a 5, donde el puntaje menor indica una baja claridad y una recomendación implícita para su reelaboración; mientras que el puntaje mayor significa una alta calidad formal. Finalmente, se le permitió a cada juez indicar las observaciones y esclarecimientos que consideraran pertinentes para el perfeccionamiento de cada reactivo. Para determinar el acuerdo entre jueces sobre la calidad de los ítems se utilizó el coeficiente V de Aiken (Aiken, 1985) y se utilizó el método score para el cálculo de los intervalos de confianza de cada coeficiente, debido a que no depende de la distribución normal de la variable y es altamente exacto (Merino \& Livia, 2009).

Para el análisis factorial exploratorio mediante el software FACTOR 10.0, se utilizó el método de extracción de mínimos cuadrados no ponderados (ULS) con rotación Promax y se estimó la consistencia interna de los factores retenidos utilizando el método $\alpha$ de Cronbach. Para el análisis factorial confirmatorio mediante el software MPlus 7 se utilizó el método de estimación de mínimos cuadrados ponderados diagonales (DWLS) y los índices de ajuste considerados fueron el estadístico chi-cuadrado $\left(\chi^{2}\right)$; el Índice de Ajuste Comparativo (CFI); el Índice de Tucker-Lewis (TLI), considerando para ambos valores entre .90 y .95 como de aceptables a excelentes; el Error Cuadrático Medio de Aproximación (RMSEA), para el que se esperan valores entre .05 y .08; y la ponderada Media Cuadrática Residual (WRMR), esperando valores menores a 1 (Yu \& Muthén, 2002). Para analizar la consistencia interna de la escala se calculó el índice de fiabilidad compuesta $(\rho)$.

Para el análisis de grupos contrastados, se realizó un análisis multivariado de la varianza (MANOVA) para analizar las diferencias en los factores y para el análisis de validez concurrente, se calcularon los coeficientes de correlación Pearson $(r)$ entre las puntuaciones del TAS-U y las medidas del DERS. Se utilizaron los criterios de Cohen (1988) para evaluar los tamaños del efecto (TE) de las correlaciones. En 
este caso se considera un TE pequeño cuando la correlación se encuentra por debajo de .10, medio cuando está en torno a .30 y grande cuando es mayor que .50 (Cohen, 1988).

\section{Resultados}

\section{Evidencia de contenido}

El análisis de la concordancia de los jueces en relación a la calidad de los ítems, dio como resultado que de los 68 reactivos evaluados, 55 presentaron coeficientes V satisfactorios superiores a .80 con intervalos de confianza entre $95 \%$ y $99 \%$. Por otro lado, 7 ítems obtuvieron coeficientes $\mathrm{V}$ de .75, los cuales fueron conservados por considerarse relevantes como indicadores del constructo; y se eliminaron 6 ítems que presentaron coeficientes menores a .75 con intervalos de confianza no significativos. Finalmente, se reformularon 11 ítems en base a las observaciones propuestas por los jueces. Como resultado, quedó una escala preliminar compuesta por 62 ítems que fueron sometidos a los análisis psicométricos recomendados por la American Educational Research Association (AERA), la American Psychological Association (APA) y el National Council of Measurement in Education (NCME) (2014).

\section{Evidencia de Estructura Interna y Análisis de Consistencia Interna}

Análisis factorial exploratorio. El índice de adecuación muestral Kaiser-Meyer-Olkin (KMO) presentó un valor de $.95 \mathrm{y}$ el test de esfericidad de Bartlett fue significativo a un nivel $p<.001$, lo cual indica una adecuada intercorrelación entre los datos y la factibilidad de realizar el análisis factorial. Utilizando el método de extracción de componentes principales y la regla de Kaiser-Gutman, se obtuvo una solución inicial de 10 factores iniciales con autovalores superiores a 1 , los cuales explicaban en conjunto un $56 \%$ de la varianza total de la prueba. Sin embargo, los resultados del análisis paralelo de Horn (1965) y del Scree Test permitieron identificar un máximo de cinco factores. Sobre la base de estos resultados, se volvió a analizar los datos solicitando la extracción de diferentes soluciones hasta cinco factores. Los resultados permitieron inferir la existencia de entre cinco y cuatro factores, observándose que la solución más clara fue la de cuatro.

A partir de la inspección de la matriz de componentes, se eliminaron los ítems con pesos factoriales bajos (menores a .40), aquellos ítems con cargas compartidas (superiores a .30) con otro factor, y aquellos ítems que no correlacionaban con ningún factor. De esta manera, se retuvieron un total de 38 ítems distribuidos en cuatro factores teóricamente claros que explicaron un $55 \%$ de la varianza de respuestas al test (Tabla 1). Tal como recomiendan Costello y Osborne (2005), cada factor identificado agrupaba al menos 5 ítems con cargas factoriales cercanas a .50. Solo se conservó un factor con cuatro ítems con cargas factoriales adecuadas (superiores a .50) por considerarlo una dimensión teóricamente relevante del constructo. Finalmente, se obtuvo una adecuada fiabilidad en todos los factores, cuyos coeficientes $\alpha$ presentaron valores considerados buenos y muy buenos (ver Tabla 1).

El examen del contenido de los ítems permitió interpretar al factor 1 como Ansiedad ante situaciones sociales con personas conocidas, el factor 2 fue denominado Ansiedad ante situaciones de desempeño académico o laboral. El factor 3 se denominó como Ansiedad a ser observado por otros en situaciones generales. Finalmente, el factor 4 fue interpretado como Ansiedad ante situaciones de abordaje afectivo-sexual.

Análisis factorial confirmatorio. El modelo inicial especificado según la estructura obtenida en el AFE, obtuvo un coeficiente $\chi^{2}$ significativo (2885.15; $p<$ .001). Los resultados mostraron índices de ajuste que, si bien no alcanzaron los valores establecidos, fueron cercanos $(C F I=.88, T L I=.87, R M S E A=.082 \mathrm{y}$ $W R M R=1.83)$. Tomando en consideración los resultados obtenidos se procedió a la reespecificación del modelo. 
Tabla 1

Matriz de configuración de los 38 ítems iniciales del TAS-U

\begin{tabular}{|c|c|c|c|c|}
\hline & \multicolumn{4}{|c|}{ Factor } \\
\hline & 1 & 2 & 3 & 4 \\
\hline & $\alpha=.92$ & $\alpha=.89$ & $\alpha=.81$ & $\alpha=.76$ \\
\hline Ítem 1 & .90 & & & \\
\hline Ítem 2 & .89 & & & \\
\hline Ítem 3 & .81 & & & \\
\hline Ítem 4 & .77 & & & \\
\hline Ítem 5 & .75 & & & \\
\hline Ítem 6 & .73 & & & \\
\hline Ítem 7 & .71 & & & \\
\hline Ítem 8 & .66 & & & \\
\hline Ítem 9 & .65 & & & \\
\hline Ítem 10 & .61 & & & \\
\hline Ítem 11 & .62 & & & \\
\hline Ítem 12 & .60 & & & \\
\hline Ítem 13 & .58 & & & \\
\hline Ítem 14 & .56 & & & \\
\hline Ítem 15 & .57 & & & \\
\hline Ítem 16 & .52 & & & \\
\hline Ítem 17 & .45 & & & \\
\hline Ítem 18 & & .90 & & \\
\hline Ítem 19 & & .88 & & \\
\hline Ítem 20 & & .88 & & \\
\hline Ítem 21 & & .82 & & \\
\hline Ítem 22 & & .67 & & \\
\hline Ítem 23 & & .63 & & \\
\hline Ítem 24 & & .49 & & \\
\hline Ítem 25 & & .47 & & \\
\hline Ítem 26 & & .43 & & \\
\hline Ítem 27 & & & .81 & \\
\hline Ítem 28 & & & .74 & \\
\hline Ítem 29 & & & .58 & \\
\hline Ítem 30 & & & .54 & \\
\hline Ítem 31 & & & .54 & \\
\hline Ítem 32 & & & .48 & \\
\hline Ítem 33 & & & .43 & \\
\hline Ítem 34 & & & .40 & \\
\hline Ítem 35 & & & & .75 \\
\hline Ítem 36 & & & & .67 \\
\hline Ítem 37 & & & & .65 \\
\hline Ítem 38 & & & & .54 \\
\hline
\end{tabular}

Nota: $\alpha=$ coeficiente de consistencia interna. 
En primer lugar, se omitieron aquellos ítems que presentaran covarianzas residuales estandarizadas altas (superiores a 2.58, según los criterios de Hair, Black, Babin, Anderson, \& Tatham, 2006), y se atendió a los índices de modificación (MI) de los mismos y a la importancia del ítem como indicador del constructo. Se obtuvo una estructura final de 27 indicadores agrupados en 4 factores (Figura 1) y con coeficientes de regresión significativos $(p<.001)$. En la reespecificación del modelo se eliminaron 11 ítems y, teniendo en cuenta los MI, se introdujeron covarianzas entre los errores de algunos ítems que pertenecían al mismo factor y que tenían un contenido equivalente (Figura 1), siguiendo las recomendaciones de Brown (2006). En relación a los índices de ajuste, si bien se obtuvo un coeficiente $\chi^{2}$ significativo, su valor se redujo considerablemente $(940.58 ; p<.001)$. Por otra parte, los índices de ajuste (CFI $=.95$, TLI $=.95$, RMSEA $=.063$ y WRMR $=1.19$ ) fueron satisfactorios por lo cual se conservó el modelo priorizando la retención de ítems teóricamente relevantes. Como se observa, la versión final de la escala resultó con menos ítems que los iniciales, sin embargo, tal como señala Uriel y Aldás (2005), en aquellos casos en los cuales la eliminación de un reactivo puede aumentar el ajuste del modelo, y tiene un sentido teórico, la eliminación puede ser deseable en términos de parsimonia.

Finalmente, se calculó la fiabilidad compuesta de las escalas, obteniendo .87 para el factor 1, .89 para el factor 2, .81 para el factor 3, y .84 para el factor 4. Se observa que todos los constructos latentes presentaron valores considerados buenos por la literatura.

Invarianza factorial. Para observar si la estructura factorial difiere según el sexo, se realizó un análisis de invariancia para el modelo configural, el modelo métrico y el modelo escalar. Los tres modelos obtuvieron índices de ajuste satisfactorios (Tabla 2). Las diferencias de $\chi^{2}$ entre el modelo de base (configural) y el modelo métrico fueron significativas.
Dado que la prueba de chi-cuadrado es sensible al tamaño de la muestra, basado en el criterio de Chen (2007), se indica que en el modelo métrico no hubo alteraciones significativas en el ajuste, ya que no hubo descenso mayor a .10 del CFI ni incremento mayor a .15 en el RMSEA. Por otro lado, las diferencias con el modelo escalar fueron no significativas por lo que se determina que la escala es invariante para ambos sexos.

\section{Análisis de grupos contrastados}

En primer lugar, se igualaron los grupos eligiendo al azar 297 casos del grupo de mujeres, quedando confeccionada la muestra final con 594 casos. En el MANOVA se obtuvieron resultados significativos en la prueba de contraste Lambda de Wilks $=.983, \mathrm{~F}$ (2.67), $p<.05$. En los contrastes post hoc sobre el impacto de la VI en las VD mediante contrastes ANOVA univariados se pudieron observar diferencias significativas $(p<.05)$ a favor de las mujeres en situaciones académicas y en situaciones de abordaje afectivo-sexual $(p<.001)$, con tamaños del efecto bajos (Tabla 3). Utilizando el procedimiento RoyBargmann Stepdown Analysis, se observaron diferencias significativas $(p<.05)$ en los mismos factores (AS en situaciones académicas y en abordaje afectivo-sexual) a favor de las mujeres y no hubo variación en el tamaño del efecto.

\section{Análisis de validez concurrente}

Los resultados de diversas investigaciones evidencian que las personas socialmente ansiosas podrían presentar un déficit específico en sus habilidades de regulación emocional (McClure \& Pine, 2015). Teniendo en cuenta esto, se correlacionaron las diferentes subescalas del TAS-U y los factores de primer y segundo orden del DERS, observándose relaciones positivas significativas (valores $r$ comprendidos entre .10 y .29). Los tamaños del efecto fueron medianos y bajos (ver Tabla 4). 


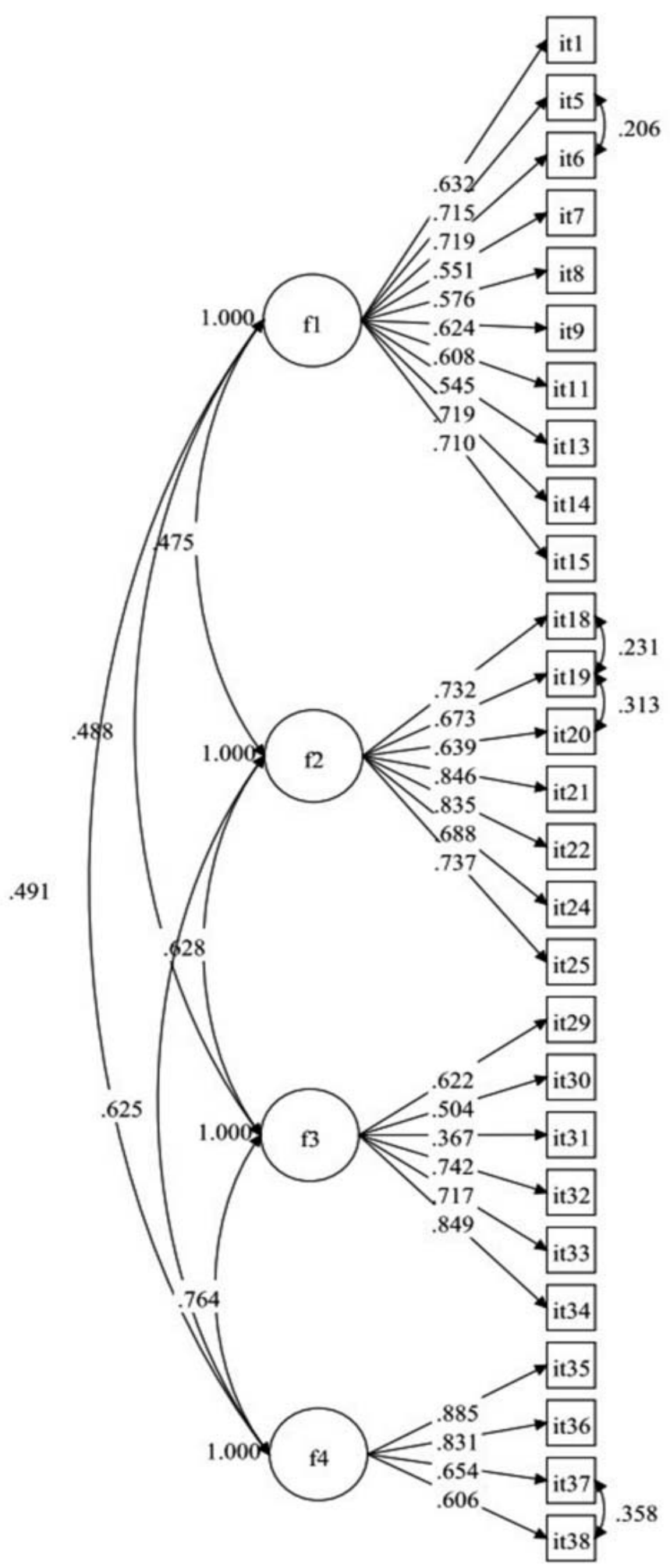

Figura 1. Modelo final de 27 ítems y 4 factores y sus coeficientes estandarizados de regresión. 
Tabla 2

Índices de ajuste en el Análisis de Invarianza Factorial según sexo

\begin{tabular}{lccccccccc}
\hline & $\chi^{2}$ & gl & CFI & TLI & RMSEA & WRMR & $\Delta \chi^{2}$ & $\Delta$ CFI & $\Delta$ RMSEA \\
\hline Configural & 1382.46 & 632 & .94 & .94 & .07 & 1.55 & & & \\
Métrica & 1228.25 & 651 & .95 & .95 & .06 & 1.51 & $154.21^{* *}$ & +.01 & -.01 \\
Escalar & 1289.01 & 894 & .95 & .96 & .04 & 1.59 & 93.45 & +.01 & -.03 \\
\hline
\end{tabular}

Nota: gl = grados de libertad; $\Delta \chi^{2}=$ diferencia en chi-cuadrado; $\Delta \mathrm{CFI}=$ diferencia en CFI.

$* * p<.001$

Tabla 3

Resultados para la prueba post hoc de Contrastes ANOVA Univariados

\begin{tabular}{lcccccc}
\hline \multirow{2}{*}{\multicolumn{1}{c}{ Factor }} & \multicolumn{2}{c}{$M(D E)$} & & & \\
\cline { 2 - 3 } & Hombre & Mujer & & Sig. & $\eta_{\mathrm{p}}{ }^{2}$ \\
\hline AS. en situaciones con conocidos & $40.83(16.13)$ & $40.18(16.61)$ & .10 & .75 & .000 \\
AS. en situaciones académicas & $40.81(14.48)$ & $43.22(13.96)$ & & 4.28 & $.04 *$ & .007 \\
AS. en situaciones generales & $27.65(11.34)$ & $28.32(11.41)$ & .51 & .48 & .001 \\
AS. en abordaje afectivo-sexual & $23.33(8.84)$ & $24.99(8.86)$ & & 5.2 & $.02 *$ & .009 \\
\hline
\end{tabular}

Nota: $* p<.05$

Tabla 4

Correlaciones entre los diferentes factores del TAS-U y dificultades en la regulación emocional

\begin{tabular}{lcccc}
\hline & $\begin{array}{c}\text { Situaciones } \\
\text { con conocidos }\end{array}$ & $\begin{array}{c}\text { Situaciones } \\
\text { académicas }\end{array}$ & $\begin{array}{c}\text { Situaciones } \\
\text { generales }\end{array}$ & $\begin{array}{c}\text { Abordaje } \\
\text { afectivo-sexual }\end{array}$ \\
\hline Falta de claridad emocional & $.11^{*}$ & $.14^{* *}$ & $.21^{* *}$ & $.12^{*}$ \\
Falta de aceptación emocional & $.23^{* *}$ & $.12^{* *}$ & $.28^{* *}$ & $.21^{* *}$ \\
Dificultades en control de impulsos & $.17^{* *}$ & $.17^{* *}$ & $.22^{* *}$ & $.15^{* *}$ \\
Interferencia en metas & $.17^{* *}$ & $.10^{*}$ & $.25^{* *}$ & $.18^{* *}$ \\
Dificultades en procesamiento emocional & $.18^{* *}$ & $.14^{* *}$ & $.28^{* *}$ & $.18^{* *}$ \\
Dificultades en regulación de respuesta emocional & $.23^{* *}$ & $.16^{* *}$ & $.29^{* *}$ & $.21^{* *}$ \\
\hline
\end{tabular}

Nota: ${ }^{*} p<.05,{ }^{* *} p<.001$ 


\section{Discusión}

La ansiedad social es una problemática de gran prevalencia en la actualidad, por lo cual profundizar en el conocimiento de este fenómeno en población universitaria resulta de suma importancia. Para contribuir a este campo de investigación, se hace imprescindible contar con instrumentos de evaluación que permitan obtener mediciones válidas y confiables. Por ello, el objetivo de este trabajo consistió en la construcción del Test de Ansiedad Social para universitarios (TAS-U).

En primer lugar, se realizó una exhaustiva revisión de las escalas de ansiedad social existentes, los desarrollos teóricos y empíricos antecedentes, y se realizaron consultas con expertos. Para el diseño de los ítems, se tuvieron en cuenta diferentes situaciones sociales, distintos números de integrantes y diversos niveles de familiaridad, y los distintos componentes de la AS que la literatura sugiere. El segundo paso fue recabar evidencia de contenido a través del juicio de expertos sobre la inclusión y calidad de los reactivos obteniéndose un instrumento inicial de 62 ítems que fueron sometidos a estudios de estructura interna mediante AFE. Este análisis permitió identificar una estructura interna compuesta de 38 ítems distribuidos en cuatro factores, que posteriormente fue confirmada mediante el AFC, aunque el número final de ítems se redujo a 27 indicadores.

El primer factor se denomina Ansiedad ante situaciones sociales con personas conocidas, el cual está presente en otros instrumentos de AS, tales como Social Anxiety Scale for adolescents (SAS-A; La Greca \& López, 1998), Social Interaction Anxiety Scale (SIAS; Heimberg et al., 1993), y Social Phobia Scale (SPS; Heimberg et al., 1999). Este factor evalúa ansiedad ante situaciones de interacción social con amigos, colegas o familiares, involucrando demandas sociales generales, como iniciar una conversación, hasta exigencias más específicas que pueden implicar una potencial evaluación por parte de los demás, por ejemplo, defender las propias ideas o hablar de sexualidad.
El segundo factor se denomina Ansiedad ante situaciones de desempeño académico o laboral, y, junto al cuarto factor, evalúa ansiedad generada ante situaciones y demandas sociales, características en los estudiantes universitarios. En particular, este factor mide ansiedad ante situaciones de demanda con diferentes niveles de exigencia que se presentan cotidianamente como parte de las actividades típicas del periodo de formación profesional (rendir exámenes o interactuar con profesores, por ejemplo). Este tipo de tareas implica instancias de evaluación real, ya sea por parte de los superiores, como también por parte de los compañeros o colegas. Del Prette y Del Prette (2003) indican que tanto el mercado laboral como los mismos estudiantes universitarios presentan una evidenciada carencia de herramientas interpersonales en sus escenarios de actuación cotidiana, y ante ello, son quienes presentan una mayor demanda de intervención y abordaje, por lo cual resulta una dimensión de suma relevancia dentro de los aspectos a ser evaluados como parte de la escala.

El tercer factor se denomina Ansiedad a ser observado por otros en situaciones generales y el mismo también ha sido evidenciado en la estructura factorial de la SIAS y en la SPS. Por otro lado, puede encontrarse su equivalente en la subescala Miedo al desempeño de la Liebowitz Social Anxiety Scale (LSAS; Heimberg et al., 1999). Este factor evalúa ansiedad generada por el miedo a ser observado por otras personas al ejecutar acciones cotidianas que no necesariamente implican una exposición o una instancia evaluativa, por ejemplo, comer o caminar. Como se ha señalado, la ansiedad social se caracteriza por miedo a situaciones sociales o desempeños en público, por temor a que resulten vergonzosas o humillantes (APA, 2000). En este sentido, quien padece ansiedad producto de las situaciones presentadas en este factor, estaría ejerciendo un automonitoreo excesivo o una hipervigilancia, característica de este trastorno (Rapee \& Heimberg, 1997).

Finalmente, el cuarto factor se denomina Ansiedad ante situaciones de abordaje afectivo-sexual. Este 
factor evalúa ansiedad generada por la interacción o abordaje hacia personas que resulten atractivas, y también fue evidenciado en la estructura factorial del Cuestionario de Ansiedad Social para Adultos (CASOA30, Caballo et al., 2010) y se considera de suma importancia su atención en la población universitaria, ya que investigaciones han demostrado la prevalencia de dificultades de los adolescentes para relacionarse con el sexo opuesto (Coronel, Levin, \& Mejail, 2011; Velázquez \& Trenas, 2014). Sumado a esto, los resultados de diferentes investigaciones evidencian que el uso de sustancias, tales como el alcohol, se convierte en una de las estrategias de afrontamiento predilectas ante la ansiedad que surge a la hora de acercarse o interactuar con otros individuos que provocan una atracción sexual y/o intereses afectivos (Buckner \& Matthews, 2012; Camacho, 2005).

Con respecto a la fiabilidad de la escala, todos los factores presentaron una consistencia interna adecuada según los criterios sugeridos por la preceptiva psicométrica en general, principalmente en los primeros estudios de construcción de una escala (Campo-Arias \& Oviedo, 2008; Roberts, Priest, \& Traynor, 2006). De acuerdo a la normativa que proponen Nunnally y Bernstein (1995), la escala permite su utilización confiable tanto para fines de investigación como para aplicaciones con fines clínicos ya que los coeficientes de fiabilidad compuesta superaron el valor de .80 en todas las subescalas.

Los resultados del estudio de validez por grupos contrastados evidencian diferencias entre hombres y mujeres, a favor del género femenino en los factores Ansiedad ante situaciones de abordaje afectivosexual y en Ansiedad ante situaciones de desempeño académico o laboral, tal como se ha evidenciado en investigaciones antecedentes (Asher et al., 2017). Estudios de diferencias de género revelan que las mujeres presentan mayores niveles de ansiedad social, especialmente ante situaciones de evaluación social real (La Greca \& López, 1998) tales como las situaciones que estas dimensiones plantean. Xu et al. (2012) observaron que la prevalencia de vida del trastorno de ansiedad social es de $4.2 \%$ para los hombres y de $5.67 \%$ para las mujeres, quienes a su vez mostraron mayor número de situaciones sociales temidas, así como también más miedos relacionados con el contexto laboral o profesional, y las consecuencias que la ansiedad podría traer a su carrera. Este mismo hallazgo fue reportado anteriormente en otro estudio (Turk et al., 1998) donde además se indica que las situaciones en este contexto que generan mayor ansiedad a las mujeres involucran hablar con personas con autoridad, presentarse ante una audiencia o hablar en público, trabajar mientras es observada, hablar en reuniones y presentar informes, expresar desacuerdo o desaprobación, ser el centro de atención, entre otras.

Por otro lado, con respecto a la ansiedad de abordaje afectivo sexual, Xu et al. (2012) encontraron que los hombres eran más propensos a padecer ansiedad ante situaciones vinculadas a proponer citas o encuentros amorosos; este dato no es coincidente con las diferencias encontradas en nuestro estudio, ya que han sido las mujeres quienes presentan mayores niveles de ansiedad en este punto. Esta divergencia podría tener su explicación en cuestiones culturales. Coincidiendo con Daros (2014) si bien en Latinoamérica se están produciendo cambios en los estereotipos y creencias de género en relación al abordaje afectivo y sexual, que incluyen una mayor toma de iniciativa de las mujeres en estas situaciones, su desempeño continúa aun siendo más discreto y selectivo que el de los hombres, y la exposición a un potencial rechazo o evaluación negativa podría tener mayores repercusiones afectivas.

Finalmente, Turk et al. (1998) encontraron que tanto hombres y mujeres presentaron niveles similares de ansiedad ante situaciones de interacción informal como, por ejemplo, ir a una fiesta, o en situaciones en general, como caminar o comer en lugares públicos. Este antecedente es coincidente con nuestros hallazgos, donde no se reportan diferencias significativas en el resto de los factores de la escala que refieren a este tipo de situaciones. 
Con respecto a la validez externa, el examen de la matriz de correlaciones entre las diferentes escalas del TAS-U y diferentes dificultades en la regulación emocional mostró relaciones significativas en la dirección esperada. Mennin, McLaughlin y Flanagan (2009) evidenciaron que las dificultades en la comprensión de las emociones es un factor predictor del diagnóstico de SAD. Sumado a ello, los resultados de otros estudios evidencian que las personas que utilizan la supresión como método de regulación emocional presentan dificultades en sus relaciones interpersonales y su competencia social dado que la menor expresividad emocional genera una respuesta negativa en las otras personas (Cutuli, 2014; English \& John, 2013; Reeck, Ames, \& Ochsner, 2016). En este sentido, en la presente investigación se observó que los universitarios con mayores niveles de ansiedad social presentan también mayores dificultades para regular sus emociones $y$, por el contrario, quienes no padecen niveles disfuncionales de ansiedad social pueden realizar un uso más adaptativo de sus estrategias de regulación emocional tal como se plantea en estudios antecedentes (Blanco \& Joormann, 2017; Jazaieri, Morrison, Goldin, \& Gross, 2015; HelbigLang, Rusch, \& Lincoln, 2015; McClure \& Pine, 2015). Tomando en consideración el modelo de déficits en el comportamiento interpersonal (Del Prette \& Del Prette, 2014), las dificultades interpersonales pueden deberse a un déficit en el repertorio o a la inhibición comportamental mediada por pensamientos y cogniciones. Así, en el caso de la ansiedad social, las dificultades en la regulación de emociones específicas, como la vergüenza, podrían mediar los comportamientos de evitación que muchas de las personas utilizan como estrategias de afrontamiento, incluyendo la supresión emocional, la evitación activa de emociones o la rumiación. Sin embargo, es necesario incrementar la investigación al respecto.

Con respecto a las limitaciones de este estudio, el método de muestreo fue de tipo no probabilístico accidental, el cual si bien puede ser utilizado cuando se trata de participantes con características heterogéneas, no se debe ignorar que conlleva un riesgo importante de que los individuos seleccionados no sean representativos de la población. No obstante, la factibilidad de aplicar métodos de muestreo probabilísticos en el conjunto estudiado, es reducida, por lo que para minimizar la probabilidad de sesgos, se tomaron ciertos recaudos tales como incluir universidades públicas y privadas, carreras de diferentes facultades y ramas de estudio, y alumnos de distintos años de cursado. Otra limitación es que no se identificaron diagnósticos de psicopatología ansiosa en los participantes, lo cual no permitió validar el funcionamiento del instrumento con muestras clínicas.

Los resultados obtenidos aportaron evidencia inicial de validez de contenido y de estructura interna, así como también evidencia de validez externa mediante análisis de concurrencia y de grupos contrastados para la evaluación de la ansiedad social en universitarios argentinos. Sin embargo, es preciso tener en cuenta que es pertinente ampliar el estudio realizando nuevos análisis que permitan obtener evidencia de otras fuentes de validez, por ejemplo, el análisis de detección de cambios ante una intervención, así como también la evaluación en poblaciones clínicas como se mencionó.

Este estudio presenta una nueva herramienta que siendo optimizada representará un importante aporte para las instituciones universitarias y sus gabinetes psicoterapéuticos, ya que puede ser incorporada en baterías de tests para identificar diferencias individuales en los estudiantes con el fin de generar programas de asistencia en la adaptación al contexto académico y en el trascurso de la vida universitaria. De esta manera, no solo se trata de una contribución al desempeño y éxito académico, sino también sería un aporte para que el paso por la universidad no solo sea una instancia de formación educacional, sino también una experiencia de aprendizaje y crecimiento personal, lo cual contribuye tanto al éxito profesional futuro, como al bienestar individual. 
Por otro lado, también resulta un instrumento de relevancia para diferentes investigaciones que pretendan continuar contribuyendo al campo de conocimiento de la ansiedad social en estudiantes, no solo incrementando los reportes epidemiológicos, sino también en la indagación sobre la interacción de la AS con otros factores.

Finalmente, con respecto a la pertinencia de utilizar esta escala en otros países sudamericanos, es importante tener en cuenta que, si bien el lenguaje empleado resulta aplicable para aquellos de habla hispana, la adecuación de un instrumento de medición no solo requiere ajustar cuestiones idiomáticas, sino también es imprescindible atender al ajuste cultural, por lo que se recomienda evaluar si el contenido es adecuado a las características culturales de la población en la cual se pretende utilizar (Lauffer, Solé, Bernstein, Lopes, \& Francisconi, 2013).

\section{Conflicto de intereses}

Los autores manifiestan que no existen conflictos de intereses de ningún tipo.

\section{Responsabilidad ética}

En esta investigación no se han realizado experimentos con humanos ni animales. Los participantes respondieron las encuestas previa aceptación del consentimiento informado. Se siguieron las normativas vigentes en el momento de la recolección de datos donde, previo a la administración de los tests, se solicita el consentimiento de los participantes, informando sobre los objetivos del trabajo, la voluntariedad de la participación, el anonimato y la confidencialidad de los datos. En este estudio, no aparecen datos personales de los participantes que permiten revelar su identidad, solo se reportan estadísticas del total de la muestra.

\section{Referencias}

Adams, G. C., Balbuena, L., Meng, X., \& Asmundson, G. J. (2016). When social anxiety and depression go together: A population study of comorbidity and associated consequences. Journal of Affective Disorders, 206, 48-54. doi: 10.1016/j.jad.2016.07.031

Agudelo-Vélez, D. A., Casadiegos-Garzón, C. P., \& Sánchez-Ortíz, D. L. (2009). Relación entre esquemas maladaptativos tempranos y características de ansiedad y depresión en estudiantes universitarios. Universitas Psychologica, 8(1), 87-104. doi: 10.1016/s0034-74 50(14)60256-0

Aiken, L. R. (1985). Three coefficients for analyzing the reliability and validity of ratings. Educational and Psychological Measurement, 45, 131-142. doi: 10.11 77/0013164485451012

American Educational Research Association, American Psychological Association, \& National Council of Measurement in Education (2014). The standards for educational and psychological testing. Washington, DC: AERA.

American Psychiatric Association (2000). Diagnostic and statistical manual of mental disorders (DSM IV) (4th ed.). Washington, DC: APA.

American Psychiatric Association (2013). Diagnostic and statistical manual of mental disorders ( $5^{\text {th }} \mathrm{ed}$.). Arlington, VA: APA.

Asher, M., Asnaani, A., \& Aderka, I. M. (2017). Gender differences in social anxiety disorder: A review. Clinical Psychology Review, 56, 1-12. doi: 10.1016/ j.cpr.2017.05.004

Bandelow, B., \& Michaelis, S. (2015). Epidemiology of anxiety disorders in the 21st century. Dialogues in Clinical Neuroscience, 17(3), 327-335.

Beidel, D. C., Turner, S. M., \& Dancu, C. V. (1985). Physiological, cognitive and behavioral aspects of social anxiety. Behaviour Research and Therapy, 23(2), 109117. doi: 10.1016/0005-7967(85)90019-1

Beiter, R., Nash, R., McCrady, M., Rhoades, D., Linscomb, M., Clarahan, M., \& Sammut, S. (2015). The prevalence and correlates of depression, anxiety, and stress in a sample of college students. Journal of Affective Disorders, 173, 90-96. doi: 10.1016/j.jad.2014.10.054 
Bhamani, S., \& Hussain, N. (2012). Social anxiety in higher education learning context: Scale construction and reliability. Golden Research Thoughts, 2(5), 1-5.

Black, J. J., Clark, D. B., Martin, C. S., Kim, K. H., Blaze, T. J., Creswell, K. G., \& Chung, T. (2015). Course of alcohol symptoms and social anxiety disorder from adolescence to young adulthood. Alcoholism: Clinical and Experimental Research, 39(6), 1008-1015. doi: 10.1111/acer.12711

Blanco, I., \& Joormann, J. (2017). Examining facets of depression and social anxiety: The relation among lack of positive affect, negative cognitions, and emotion dysregulation. The Spanish Journal of Psychology, 20, E51. doi: 10.1017/sjp.2017.43

Brown, T. A. (2014). Confirmatory factor analysis for applied research. New York: Guilford Publications.

Brook, C. A., \& Willoughby, T. (2015). The social ties that bind: Social anxiety and academic achievement across the university years. Journal of Youth and Adolescence, 44(5), 1139-1152. doi: 10.1007/ s10964-015-0262-8

Buckner, J. D., \& Matthews, R. A. (2012). Social impressions while drinking account for the relationship between alcohol-related problems and social anxiety. Addictive Behaviors, 37, 533-536. doi: 10.1016/j.addb eh.2011.11.026

Caballo, V. E., Salazar, I. C., Arias, B., Irurtia, M., Calderero, M., \& CISO-A Research Team. (2010). Validación del Cuestionario de Ansiedad Social para Adultos (CASOA30) en universitarios españoles: similitudes y diferencias entre carreras universitarias y comunidades autónomas. Behavioral Psychology, 18(1), 5-34.

Camacho, I. (2005). Consumo de alcohol en universitarios: relación funcional con los factores sociodemográficos, las expectativas y la ansiedad. Acta Colombiana de Psicología, 8(1), 91-120.

Campo-Arias, A., \& Oviedo, H. C. (2008). Propiedades psicométricas de una escala: la Consistencia Interna. Revista De Salud Pública, 10, 831-839.

Chen, F. F. (2007). Sensitivity of goodness of fit indexes to lack of measurement invariance. Structural Equation Modeling: A Multidisciplinary Journal, 14(3), 464-504. doi: 10.1080/10705510701301834
Cohen, J. (1988). Statistical power analysis for the behavioral sciences (2nd ed.). Hillsdale, NJ: Erlbaum.

Coker, A. L., Fisher, B. S., Bush, H. M., Swan, S. C., Williams, C. M., Clear, E. R., \& DeGue, S. (2015). Evaluation of the Green Dot bystander intervention to reduce interpersonal violence among college students across three campuses. Violence against Women, 21(12), 1507-1527. doi: 10.1177/107780121454 5284

Collins, A. B. (2009). Investigation of social anxiety prevalence and anxiety sensitivity among college students. Universidad de Nevada, EE.UU.

Coronel, C. P., Levin, M., \& Mejail, S. (2011). Social skills: An investigation with young adolescents from different socioeconomic contexts. Electronic Journal of Research in Educational Psychology, 9(1), 241262.

Costello, A. B., \& Osborne, J. W. (2005). Best practices in exploratory factor analysis: Four recommendations for getting the most from your analysis. Assessment, 10(7), 1-9. doi: 10.4135/9781412995627.d8

Cummings, C. M., Caporino, N. E., \& Kendall, P. C. (2014). Comorbidity of anxiety and depression in children and adolescents: 20 years after. Psychological Bulletin, 140(3), 816-845. doi: 10.1037/a0034733

Cutuli, D. (2014). Cognitive reappraisal and expressive suppression strategies role in the emotion regulation: an overview on their modulatory effects and neural correlates. Frontiers in Systems Neuroscience, 8, 175. doi: 10.3389/fnsys.2014.00175

Daros, W. R. (2014). La mujer posmoderna y el machismo. Franciscanum, 56(162), 107-129.

Delgado, B., Inglés, C. J., \& García-Fernández, J. M. (2013). Social anxiety and self-concept in adolescence. Revista de Psicodidáctica, 18(1), 179194. doi: 10.1387/RevPsicodidact.6411

Del Prette, A., \& Del Prette, Z. A. P. (2014). Psicologia das relações interpessoais: Vivências para o trabalho em grupo (11th Ed.). São Paulo: Ed. Vozes.

Del Prette, Z. A. P., \& Del Prette, A. (2003). No contexto da travessia para o ambiente de trabalho: Treinamento de habilidades sociais com universitários. Estudos de Psicología, 8(3), 413-420. 
Eysenck, M. W. (2013). Anxiety: The cognitive perspective. London: Psychology Press.

English, T., \& John, O. P. (2013). Understanding the social effects of emotion regulation: The mediating role of authenticity for individual differences in suppression. Emotion, 13(2), 314-329. doi:10.1037/ a0029847

Fernández-Sogorb, A., Aparicio-Flores, M., Granados, L., Aparisi-Sierra, D., \& Inglés, C. J. (2012). Ansiedad social y fobia social: revisión de autoinformes y análisis de su fiabilidad y validez en muestra infantojuvenil española. Calidad de Vida y Salud, 11(1), 30-36.

Galli, A. C. (2005). Prevalencia de trastornos psicopatológicos en alumnos de Psicología. Revista de Psiquiatría y Salud Mental Hermilio Valdizan, 6(1), 55-66.

Gratz, K. L., \& Roemer, L. (2004). Multidimensional assessment of emotion regulation and dysregulation: Development, factor structure, and initial validation of the Difficulties in Emotion Regulation Scale. Journal of Psychopathology and Behavioral Assessment, 26(1), 41-54. doi: 10.1023/B:JOBA.0000007455.08539.94

Gross, J. J., \& Jazaieri, H. (2014). Emotion, emotion regulation, and psychopathology an affective science perspective. Clinical Psychological Science, 2(4), 387-340. doi: 10.1177/2167702614536164

Jazaieri, H., Morrison, A. S., Goldin, P. R., \& Gross, J. J. (2015). The role of emotion and emotion regulation in social anxiety disorder. Current Psychiatry Reports, 1(17), 1-9. doi: 10.1007/s11920-014-0531-3

Hair, J. F., Black, W. C., Babin, B. J., Anderson, R. E., \& Tatham, R. L. (2006). Multivariate data analysis (6th ed.). New Jersey: Pearson Education.

Ham, L. S., Connolly, K. M., Milner, L. A., Lovett, D. E., \& Feldner, M. T. (2013). Substance abuse and anxiety disorders: The case of social anxiety disorder and PTSD. In E. A. Storch \& D. McKay (Eds.), Handbook of Treating Variants and Complications in Anxiety Disorders (pp. 285-306). New York: Springer Science. doi: 10.1007/978-1-4614-6458-7_18

Heimberg, R. G., Horner, K. J., Juster, H. R., Safren, S. A., Brown, E. J., Schneier, F. R., \& Liebowitz, M. R.
(1999). Psychometric properties of the Liebowitz Social Anxiety Scale. Psychological Medicine, 29(1), 199-212.

Heimberg, R. G., Mueller, G. P., Holt, C. S., Hope, D. A., \& Liebowitz, M. R. (1993). Assessment of anxiety in social interaction and being observed by others: The Social Interaction Anxiety Scale and the Social Phobia Scale. Behavior Therapy, 23(1), 53-73. doi: 10.1016/ S0005-7894(05)80308-9

Helbig Lang, S., Rusch, S., \& Lincoln, T. M. (2015). Emotion regulation difficulties in social anxiety disorder and their specific contributions to anxious responding. Journal of Clinical Psychology, 71(3), 241-249. doi: 10.1002/jclp.22135

Horn, J. (1965). A rationales and test for the number of factor in factor analysis. Psychometrika, 30(2), 179185. doi: $10.1007 / \mathrm{bf02289447}$

Kaukinen, C. (2014). Dating violence among college students: The risk and protective factors. Trauma, Violence, \& Abuse, 15(4), 283-296. doi: 10.1177/ 1524838014521321

La Greca, A. M., \& López, N. (1998). Social anxiety among adolescents: Linkages with peer relations and friendships. Journal of Abnormal Child Psychology, 26(2), 83-94.

Lauffer, A., Solé, L., Bernstein, S., Lopes, M. H., \& Francisconi, C. F. (2013). Cómo minimizar errores al realizar la adaptación transcultural y la validación de los cuestionarios sobre calidad de vida: aspectos prácticos. Revista de Gastroenterología de México, 78(3), 159176. doi: 10.1016/j.rgmx.2013.01.008

Leary, M. R., \& Kowalski, R. M. (1995). Social anxiety. New York: The Guilford Press.

Mcclure, E. B., \& Pine, D. S. (2015). Social anxiety and emotion regulation: A model for developmental psychopathology perspectives on anxiety disorders. Developmental Psychopathology, 470-502. doi: 10.10 02/9780470939406.ch12

Medrano, L. A., \& Trogolo, M. (2016). Construct validity of the difficulties in emotion regulation scale: Further evidence using confirmatory factor analytic approach. Abnormal and Behavioural Psychology, 2(2). doi: 10. 4172/2472-0496.1000117 
Mennin, D. S., McLaughlin, K. A., \& Flanagan, T. J. (2009). Emotion regulation deficits in generalized anxiety disorder, social anxiety disorder, and their cooccurrence. Journal of Anxiety Disorders, 23(7), 866-871. doi: 10.1016/j.janxdis.2009.04.006

Merino, C., \& Livia, J. (2009). Intervalos de confianza asimétricos para el índice la validez de contenido: Un programa Visual Basic para la V de Aiken. Anales de Psicología, 25(1), 169-171.

Montero, I., \& León, O. G. (2002). Clasificación y descripción de las metodologías de investigación en Psicología. International Journal of Clinical and Health Psychology, 2(3), 503-508.

Morán, V. E., Olaz, F. O., \& Del Prette, Z. A. P. (2015). Social Skills Questionnaire for Argentinean college students (SSQ-U). Development and validation. The Spanish Journal of Psychology, 18, E95. doi:10.1017/ sjp.2015.92

Morán, V. E., Olaz, F. O., Pérez, E. R., \& Del Prette, Z. A. P. (2018). Emotional-evolutional model of social anxiety in university students. International Journal of Psychology and Psychological Therapy, 18(3), 315-330.

Nicholls, J., Staiger, P. K., Williams, J. S., Richardson, B., \& Kambouropoulos, N. (2014). When social anxiety co-occurs with substance use: Does an impulsive social anxiety subtype explain this unexpected relationship? Psychiatry Research, 220(3), 909-914. doi: 10.1016/j.psychres.2014.08.040

Nunnally, J. C., \& Bernstein, I. J. (1995). Teoría psicométrica. Madrid: McGraw-Hill

Parray, W. M., \& Kumar, S. (2016). Assertiveness among undergraduate students of the university. The International Journal of Indian Psychology, 4(1), 283-291. doi: 18.01.021/20160476

Rana, S. A., Akhtar, S., \& Tahir, M. A. (2013). Parenting styles and social anxiety among adolescents. New Horizons, 7(2), 21-34.

Raposa, E. B., \& Hammen, C. (2018). A daily diary investigation of the influence of early family adversity on social functioning during the transition to adulthood. Social Development, 27(2), 431-446. doi: 10.1111/ sode.12269
Rapee, R. M., \& Heimberg, R. G. (1997). A cognitivebehavioral model of anxiety in social phobia. Behaviour research and therapy, 35(8), 741-756. doi: 10.1016/S0005-7967(97)00022-3

Rapee, R. M., \& Spence, S. H. (2004). The etiology of social phobia: Empirical evidence and an initial model. Clinical Psychology Review, 24, 737-767. doi: 10.101 6/j.cpr.2004.06.004

Reeck, C., Ames, D. R., \& Ochsner, K. N. (2016). The social regulation of emotion: An integrative, crossdisciplinary model. Trends in Cognitive Sciences, 20(1), 47-63. doi: 10.1016/j.tics.2015.09.003

Ricker, S., Ridgeway, P., Eichele, A., Perry, L., \& Zimansky, T. (2018, junio). Shyness in College Students: Changes over time and other correlates. Póster presentado en el Simposio Universitario de Investigación, Illinois, Estados Unidos, 130. Recuperado de https://ir.library.illinoisstate.edu/ rsp_urs/130

Roberts, P., Priest, H., \& Traynor, M. (2006). Reliability and validity in research. Nursing Standard, 20(44), 41-45. doi:10.7748/ns2006.07.20.44.41.c6560

Salazar, I. C., Caballo, V. E., \& Arias, B. (2016). Validez de constructo y fiabilidad del «Cuestionario de Ansiedad Social para Adultos» (CASO) en Colombia. Revista Latinoamericana de Psicología, 48(2), 98107. doi: 10.1016/j.rlp.2015.07.001

Turner, S. M., Stanley, M. A., Beidel, D. C., \& Bond, L. (1989). The social phobia and anxiety inventory: construct validity. Journal of Psychopathology and Behavioral Assessment, 11(3), 221-234. doi: 10.100 7/BF00960494

Turk, C. L., Heimberg, R. G., Orsillo, S. M., Holt, C. S., Gitow, A., Street, L. L., Schneier, F. R., \& Liebowitz, M. R. (1998). An investigation of gender differences in social phobia. Journal of Anxiety Disorders, 12(3), 209-223.

Uriel, E., \& Aldás, J. (2005). Análisis Multivariante Aplicado. España: Thomson.

Velásquez, C., Montgomery, U. W., Montero, L. V., Pomalaya, V. R., Dioses, C. A., Velásquez, N. C. A., Araki, O. R., \& Reynoso, E. D. (2008). Bienestar psicológico, asertividad y rendimiento académico en 
estudiantes universitarios sanmarquinos. Revista Investigación Psicológica, 11(2), 139-152. doi: 10.15 381/rinvp.v11i2.3845

Velázquez, M. E. C., \& Trenas, A. F. R. (2014). Estilos educativos parentales y su relación con la socialización en adolescentes. Apuntes de Psicología, 32(3), 271280.

Walters, K. S., Bulmer, S. M., Troiano, P. F., Obiaka, U., \& Bonhomme, R. (2018). Substance use, anxiety, and depressive symptoms among college students. Journal of Child \& Adolescent Substance Abuse, 27(2), 103111. doi: 10.1080/1067828x.2017.1420507

Wong, Q. J., \& Rapee, R. M. (2016). The aetiology and maintenance of social anxiety disorder: A synthesis of complimentary theoretical models and formulation of a new integrated model. Journal of Affective Disorders, 203, 84-100. doi: 10.1016/j.jad.2016.05. 069

Xu, Y., Schneier, F., Heimberg, R. G., Princisvalle, K., Liebowitz, M. R., Wang, S., \& Blanco, C. (2012). Gender differences in social anxiety disorder: Results from the national epidemiologic sample on alcohol and related conditions. Journal of Anxiety Disorders, 26(1), 12-19. doi: 10.1016/j.janxdis.2011.08.006

Yu, C. Y., \& Muthén, B. (2002). Evaluation of model fit indices for latent variable models with categorical and continuous outcomes (Technical report). Los Angeles: UCLA, Graduate School of Education \& Information Studies.

\section{Valeria E. Moran}

Instituto de Investigaciones Psicológicas (IIPsi) CONICET- UNC. Facultad de Psicología, Universidad Nacional de Córdoba, Argentina

Doctora en Psicología, cuenta con investigaciones en comportamiento interpersonal en estudiantes universitarios y ansiedad social. Su línea de formación se focaliza en la construcción y adaptación de instrumentos de medición.

ORCID: 0003-3628-1636

Autor corresponsal: moranvaleria@gmail.com

\section{Fabián O. Olaz}

Facultad de Psicología, Universidad Nacional de Córdoba, Argentina

Doctor en Psicología. Es Peer-Reiwed ACT Trainer por la ACBS (Association for Contextual Behavioral Science). Profesor en la Facultad de Psicología, Universidad Nacional de Córdoba. Especialista en ACT y FAP con especialidad en el modelo Matrix.

ORCID: 0000-0003-2713-7413

fabidelarenta@gmail.com

\section{Edgardo R. Pérez}

Facultad de Psicología, Universidad Nacional de Córdoba, Argentina

Doctor en Psicología, cuenta con investigaciones sobre la educación del talento en adolescentes, especialmente el talento verbal y las estrategias de lectura y escritura. Su línea de formación se centra en el desarrollo y la adaptación de pruebas de intereses vocacionales y autoeficacia.

edrapester@gmail.com

\section{Zilda A. P. Del Prette}

Departamento de Psicología, Universidade Federal de São Carlos, Brasil

Doctora en Psicología. Profesora Titular de la Universidade Federal de São Carlos. Cuenta con estudios sobre el comportamiento interpersonal, siendo uno de los principales referentes en investigación sobre habilidades sociales en Latinoamérica.

ORCID: 0000-0002-0130-2911

zdprette@ufscar.br 